\title{
Results on Complex Partial b-Metric Space with an Application
}

\author{
Gunaseelan Mani $\mathbb{D}^{1}{ }^{1}$ Arul Joseph Gnanaprakasam, ${ }^{2}$ Ramakrishnan Kalaichelvan, ${ }^{1}$ \\ and Yaé Ulrich Gaba $\mathbb{D}^{3,4}$ \\ ${ }^{1}$ Department of Mathematics, Sri Sankara Arts and Science College(Autonomous), Affiliated to Madras University, Enathur, \\ Kanchipuram 631 561, Tamil Nadu, India \\ ${ }^{2}$ Department of Mathematics, College of Engineering and Technology, Faculty of Engineering and Technology, \\ SRM Institute of Science and Technology, SRM Nagar Kattankulathur, Kanchipuram, Chennai 603203, Tamil Nadu, India \\ ${ }^{3}$ Department of Mathematics and Applied Mathematics, Sefako Makgatho Health Sciences University, Ga-Rankuwa, \\ South Africa \\ ${ }^{4}$ African Center for Advanced Studies, P.O. Box 4477, Yaounde, Cameroon
}

Correspondence should be addressed to Gunaseelan Mani; mathsguna@yahoo.com and Yaé Ulrich Gaba; yaeulrich.gaba@ gmail.com

Received 2 February 2021; Revised 22 February 2021; Accepted 25 March 2021; Published 10 April 2021

Academic Editor: Stefania Tomasiello

Copyright (c) 2021 Gunaseelan Mani et al. This is an open access article distributed under the Creative Commons Attribution License, which permits unrestricted use, distribution, and reproduction in any medium, provided the original work is properly cited.

In this paper, we prove a fixed point theorem in complex partial $b$-metric space under new contraction mapping. The proved results generalize and extend some of the well-known results in the literature. We also give some applications of our main results.

\section{Introduction}

Introduced in 1989 by Bakhtin [1] and Czerwik [2], the concept of $b$-metric spaces provided a framework to extend the results already known in the classical setting of metric spaces. About two decades later, more precisely in 2011, Azamet al. [3] came up with the notion of complex-valued metric spaces and provided some common fixed point theorems under some contractive conditions. Two years after, it was in [4] Rao et al. discussed for the first time the idea of complex-valued $b$-metric spaces.

It was just very recently, in 2017, that Dhivya and Marudai [5] extended all the preceding results in the setting of complex partial metric spaces making use of a rational type contraction.

This was followed by Gunaseelan [6], who introduced the concepts of complex partial $b$-metric spaces and discussed some results of fixed point theory for self-mappings in these new spaces.

Many authors have studied related interesting metric such as structures along with some applications. And, in this line, significant results have been obtained and can be read in [7-23]. In this paper, under new contraction condition, we prove a fixed point theorem in complex partial $b$-metric space. Although there have been a significant amount of scientific contributions to the theory of partial $b$-metric space, very few address that of complex valued, and even less, the applicability of complex partial $b$-metrics in the resolution integral equations. This, however, in one the main contributions of the present work. We begin by recalling basic facts about complex partial $b$-metric spaces.

\section{Preliminaries}

Let $\mathbb{C}$ be the set of complex numbers and $\Phi_{1}, \Phi_{2}, \Phi_{3} \in \mathbb{C}$. Define a partial order $\prec$ on $\mathbb{C}$ as follows: $\Phi_{1} \prec \Phi_{2}$ if and only if Re.part $\left(\Phi_{1}\right) \leq \operatorname{Re} \cdot \operatorname{part}\left(\Phi_{2}\right), \operatorname{Im} \cdot \operatorname{part}\left(\varpi_{1}\right) \leq \operatorname{Im} \cdot \operatorname{part}\left(\varpi_{2}\right)$.

Consequently, one can infer that $\Phi_{1} \prec \Phi_{2}$ if one of the following conditions is satisfied:

(i) Re.part $\left(\Phi_{1}\right)=\operatorname{Re} \cdot \operatorname{part}\left(\Phi_{2}\right)$ and Im.part $\left(\Phi_{1}\right)<\operatorname{Im}$.part $\left(\omega_{2}\right)$ 
(ii) Re.part $\left(\Phi_{1}\right)<\operatorname{Re} \cdot \operatorname{part}\left(\Phi_{2}\right)$ and Im.part $\left(\Phi_{1}\right)=\operatorname{Im}$.part $\left(\omega_{2}\right)$

(iii) Re.part $\left(\Phi_{1}\right)<$ Re.part $\left(\Phi_{2}\right)$ and Im.part $\left(\Phi_{1}\right)<$ Im.part $\left(\omega_{2}\right)$

(iv) Re.part $\left(\Phi_{1}\right)=\operatorname{Re} \cdot \operatorname{part}\left({\Phi_{2}}_{2}\right)$ and Im.part $\left(\Phi_{1}\right)=\operatorname{Im} \cdot \operatorname{part}$ $\left(\omega_{2}\right)$

In particular, we write $\varpi_{1} \precsim \varpi_{2}$ if $\omega_{1} \neq \Phi_{2}$ and one of (i), (ii), and (iii) is satisfied, and we write $\varpi_{1} \prec \varpi_{2}$ if only (iii) is satisfied. Notice that

(a) If $0<\varpi_{1} \precsim \varpi_{2}$, then $\left|\varpi_{1}\right|<\left|\varpi_{2}\right|$

(b) If $\Phi_{1} \prec \Phi_{2}$ and $\Phi_{2} \prec \omega_{3}$, then $\Phi_{1} \prec \varpi_{3}$

(c) If $\tau, \gamma \in \mathbb{R}$ and $\tau \leq \gamma$, then $\tau \Phi_{1} \prec \gamma \Phi_{1}$ for all $0 \prec \Phi_{1} \in \mathbb{C}$

Definition 1 (see [4]). Let $\Theta$ be a nonvoid set and let $s \geq 1$ be a given real number. A function $d: \Theta \times \Theta \longrightarrow \mathbb{C}$ is called a complex-valued $b$-metric on $\Theta$ if, for all $e, f, g \in \Theta$, the following conditions are satisfied:
(i) $0 \prec d(e, f)$ and $d(e, f)=0$ if and only if $e=f$
(ii) $d(e, f)=d(f, g)$
(iii) $d(e, f) \prec s[d(e, g)+d(g, f)]$

The pair $(\Theta, d)$ is called a complex-valued $b$-metric space.

Here, $\mathbb{C}^{+}\left(=\left\{(e, g) \mid e, g \in \mathbb{R}^{+}\right\}\right)$and $\mathbb{R}^{+}(=\{e \in \mathbb{R} \mid e \geq 0\})$ denote the set of nonnegative complex numbers and the set of nonnegative real numbers, respectively. We now give the complex partial metric space.

Definition 2 (see [5]). A complex partial metric on a nonvoid set $\Theta$ is a function $\xi_{c}: \Theta \times \Theta \longrightarrow \mathbb{C}^{+}$such that, for all $\alpha, \beta, \gamma \in \Theta$,

(i) $0 \prec \xi_{c}(\alpha, \alpha) \prec \xi_{c}(\alpha, \beta)$ (small self - distances)

(ii) $\xi_{c}(\alpha, \beta)=\xi_{c}(\beta, \alpha)$ (symmetry)

(iii) $\xi_{c}(\alpha, \alpha)=\xi_{c}(\alpha, \beta)=\xi_{c}(\beta, \beta)$ if and only if $\alpha=\beta$ (equality)

(iv)

$\xi_{c}(\alpha, \beta) \prec \xi_{c}(\alpha, \gamma)+\xi_{c}(\gamma, \beta)-\xi_{c}(\beta, \beta)$ (triangularity)

A complex partial metric space is a pair $\left(\Theta, \xi_{c}\right)$ such that $\Theta$ is a nonvoid set and $\xi_{c}$ is the complex partial metric on $\Theta$.

Definition 3 (see [6]). A complex partial $b$-metric on a nonvoid set $\Theta$ is a function $\vee_{c b}: \Theta \times \Theta \longrightarrow \mathbb{C}^{+}$such that, for all $\alpha, \beta, \gamma \in \Theta$,

(i) $0 \prec \vee_{c b}(\alpha, \alpha) \prec \bigvee_{c b}(\alpha, \beta)$ (small self - distances)

(ii) $\bigvee_{c b}(\alpha, \beta)=\bigvee_{c b}(\beta, \alpha)$ (symmetry)

(iii) $\bigvee_{c b}(\alpha, \alpha)=\bigvee_{c b}(\alpha, \beta)=\bigvee_{c b}(\beta, \beta) \Leftrightarrow \alpha=\beta$ (equality)

(iv) $\exists$ a real number $s \geq 1$ and $s$ is an independent of $\alpha, \beta, \gamma$ such that $\vee_{c b}\left((\alpha, \beta) \prec s\left[\vee_{c b}(\alpha, \gamma)+\vee_{c b}(\gamma, \beta)\right]\right.$ $-\vee_{c b}(\gamma, \gamma)$ (triangularity)
A complex partial $b$-metric space is a pair $\left(\Theta, \vee_{c b}\right)$ such that $\Theta$ is a nonvoid set and $\vee_{c b}$ is the complex partial $b$-metric on $\Theta$. The number $s$ is called the coefficient of $\left(\Theta, \vee_{c b}\right)$.

Remark 1 (see [6]). In a complex partial $b$-metric space $\left(\Theta, \vee_{c b}\right)$ if $\alpha, \beta \in \Theta$ and $\vee_{c b}(\alpha, \beta)=0$, then $\alpha=\beta$, but the converse may not be true.

Every complex partial $b$-metric $\vee_{c b}$ on a nonvoid set $\Theta$ generates a topology $\tau_{c b}$ on $\Theta$ whose base is the family of open $\curlyvee_{c b}$-balls $B_{\curlyvee_{c b}}(\alpha, \varepsilon)$, where $\tau_{c b}=\left\{B_{\vee_{c b}}(\alpha, \varepsilon): \alpha \in \Theta\right.$, $\varepsilon>0\} \quad$ and $\quad B_{\vee_{c b}}(\alpha, \varepsilon)=\left\{\beta \in \Theta: \vee_{c b}(\alpha, \beta)<\varepsilon+\vee_{c b}(\alpha, \alpha)\right\}$. Now, we define Cauchy sequence and convergent sequence in complex partial $b$-metric spaces.

Definition 4 (see [6]). Let $\left(\Theta, \vee_{c b}\right)$ be a complex partial $b$-metric space with coefficient $s$. Let $\left\{\alpha_{n}\right\}$ be any sequence in $\Theta$ and $\alpha \in \Theta$. Then,

(i) The sequence $\left\{\alpha_{n}\right\}$ is said to be convergent with respect to $\tau_{c b}$ and converges to $\alpha$ if $\lim _{n \longrightarrow \infty} \vee_{c b}$ $\left(\alpha_{n}, \alpha\right)=\vee_{c b}(\alpha, \alpha)$

(ii) The sequence $\left\{\alpha_{n}\right\}$ is said to be Cauchy sequence in $\left(\Theta, \vee_{c b}\right)$ if $\lim _{n, m \rightarrow \infty} \vee_{c b}\left(\alpha_{n}, \alpha_{m}\right)$ exists and is finite

(iii) $\left(\Theta, \vee_{c b}\right)$ is said to be a complete complex partial $b$-metric space if, for every Cauchy sequence $\left\{\alpha_{n}\right\}$ in $\Theta$, there exists $\alpha \in \Theta$ such that $\lim _{n, m \rightarrow \infty} \vee_{c b}\left(\alpha_{n}\right.$, $\left.\alpha_{m}\right)=\lim _{n \longrightarrow \infty} \bigvee_{c b}\left(\alpha_{n}, \alpha\right)=\bigvee_{c b}(\alpha, \alpha)$

(iv) A mapping $\Xi: \Theta \longrightarrow \Theta$ is said to be continuous at $\alpha_{0} \in \Theta$ if, for every $\varepsilon>0$, there exists $\delta>0$ such that $\Xi\left(B_{\curlyvee_{c b}}\left(\alpha_{0}, \delta\right)\right) \subset B_{\curlyvee_{c b}}\left(\Xi\left(\alpha_{0}, \varepsilon\right)\right)$

Let $\left(\Theta, \vee_{c b}\right)$ be a complex partial $b$-metric space and $D \subseteq \Theta$. A point $\alpha \in \Theta$ is called an interior of set a $D$ if there exists $0<r \in \mathbb{C}$ such that $D_{\bigvee_{c b}}(\alpha, r)=\left\{\beta \in \Theta: \bigvee_{c b}(\alpha, \beta)<\bigvee_{c b}\right.$ $(\alpha, \alpha)+r\} \subseteq D$. A subset $D$ is called open if each point of $D$ is an interior point of $D$. A point $\alpha \in \Theta$ is said to be a limit point of $D$, for every $0<r \in \mathbb{C}, D_{\bigvee_{c b}}(\alpha, r) \cap(D-\{\alpha\}) \neq \phi$. A subset $E \subseteq \Theta$ is called closed iff $E$ contains all its limit points.

Example 1 (see [6]). Let $\Theta=[0, \infty)$ be endowed with complex partial $b$-metric $\vee_{c b}: \Theta \times \Theta \longrightarrow \mathbb{C}^{+}$with $\vee_{c b}=(\max \{\alpha, \beta\})^{2}+$ $i(\max \{\alpha, \beta\})^{2} \forall \alpha, \beta \in \Theta$.

In 2019, Gunaseelan [6] proved the following theorem.

Theorem 1 (see [6]). Let $\left(\Theta, \vee_{c b}\right)$ be a complete complex partial $b$-metric space with coefficient $s \geq 1$ and $\Xi: \Theta \longrightarrow \Theta$ be a mapping satisfying the following condition:

$$
\vee_{c b}(\Xi \alpha, \Xi \beta) \prec a\left[\vee_{c b}(\alpha, \Xi \alpha)+\vee_{c b}(\beta, \Xi \beta)\right], \quad \forall \alpha, \beta \in \Theta,
$$

where $a \in[0,(1 / s))$. Then, $\Xi$ has a unique fixed point $\gamma \in \Theta$ and $\vee_{c b}(\gamma, \gamma)=0$. 
Inspired by Theorem 1, we prove a fixed point theorem on complex partial $b$-metric space under new contraction mapping.

In Section 3, we first prove, under new contraction mapping, a fixed point theorem on complete complex partial $b$-metric space. We also provide an example of the complete complex partial $b$-metric space and clarify that, under certain conditions, it has a unique fixed point.

\section{Main Results}

Theorem 2. Let $\left(\Theta, \vee_{c b}\right)$ be a complete complex partial $b$-metric space with constant $s \geq 1$ and let $\Xi$ be a self-mapping on $\Theta$. Suppose that there exist functions $\tau_{i}, i=1,2,3,4,5$, of $\mathbb{C}^{+}$into $\mathbb{C}^{+}$such that

$\left(C_{1}\right)$ Each $\tau_{i}$ is upper semicontinuous from the right

$\left(C_{2}\right) \quad \tau_{1}(v)+\tau_{2}(v)+\tau_{3}(v)+\tau_{4}(v)+\tau_{5}(v) \prec\left(1 / 2 s^{2}\right) v$, $\forall v>0$

$\left(C_{3}\right)$ For any distinct $\alpha, \beta \in \Theta$,

$$
\begin{aligned}
& \frac{1}{2 s} \curlyvee_{c b}(\alpha, \Xi \alpha)\left\langle\bigvee_{c b}(\alpha, \beta) \Rightarrow\right. \\
& \vee_{c b}(\alpha, \beta) \vee_{c b}(\Xi \alpha, \Xi \beta) \prec \tau_{1}\left(\vee_{c b}(\alpha, \beta)\right) \vee_{c b}(\alpha, \beta) \\
& +\tau_{2}\left(\vee_{c b}(\alpha, \beta)\right) \vee_{c b}(\alpha, \Xi \beta) \\
& +\tau_{3}\left(\vee_{c b}(\alpha, \beta)\right) \vee_{c b}(\Xi \alpha, \beta) \\
& +\tau_{4}\left(\vee_{c b}(\alpha, \beta)\right) \vee_{c b}(\alpha, \Xi \alpha) \\
& +\tau_{5}\left(\vee_{c b}(\alpha, \beta)\right) \vee_{c b}(\beta, \Xi \beta)
\end{aligned}
$$

Then, $\Xi$ has a unique fixed point.

Proof. Let us first prove that if fixed points of $\Xi$ exists, then it is unique. Let $\alpha, \beta \in \Theta$ be two distinct fixed points of $\Xi$, that is, $\Xi \alpha=\alpha \neq \beta=\Xi \beta$. Therefore, $\vee_{c b}(\alpha, \beta)>0$. If $\vee_{c b}(\alpha, \alpha)$ $=0$, we have $0=(1 / 2 s) \vee_{c b}(\alpha, \alpha)=(1 / 2 s) \vee_{c b}(\alpha, \Xi \alpha) \prec \vee_{c b}(\alpha$, $\beta)$. If $\vee_{c b}(\alpha, \alpha)>0$. By the definition of complex partial $b$-metric space, we obtain

$$
\frac{1}{2 s} \curlyvee_{c b}(\alpha, \Xi \alpha)=\frac{1}{2 s} \curlyvee_{c b}(\alpha, \alpha) \prec \curlyvee_{c b}(\alpha, \beta)
$$

From condition $\left(C_{3}\right)$, we derive

$$
\begin{aligned}
& \bigvee_{c b}(\alpha, \beta)^{2}=\bigvee_{c b}(\alpha, \beta) \bigvee_{c b}(\Xi \alpha, \Xi \beta) \\
& \preccurlyeq \tau_{1}\left(\vee_{c b}(\alpha, \beta)\right) \vee_{c b}(\alpha, \beta)+\tau_{2}\left(\vee_{c b}(\alpha, \beta)\right) \vee_{c b}(\alpha, \Xi \beta) \\
& +\tau_{3}\left(\vee_{c b}(\alpha, \beta)\right) \vee_{c b}(\Xi \alpha, \beta)+\tau_{4}\left(\vee_{c b}(\alpha, \beta)\right) \vee_{c b}(\alpha, \Xi \alpha) \\
& +\tau_{5}\left(\vee_{c b}(\alpha, \beta)\right) \vee_{c b}(\beta, \Xi \beta) \\
& =\tau_{1}\left(\bigvee_{c b}(\alpha, \beta)\right) \bigvee_{c b}(\alpha, \beta)+\tau_{2}\left(\bigvee_{c b}(\alpha, \beta)\right) \bigvee_{c b}(\alpha, \beta) \\
& +\tau_{3}\left(\vee_{c b}(\alpha, \beta)\right) \vee_{c b}(\alpha, \beta)+\tau_{4}\left(\vee_{c b}(\alpha, \beta)\right) \vee_{c b}(\alpha, \alpha) \\
& +\tau_{5}\left(\vee_{c b}(\alpha, \beta)\right) \vee_{c b}(\beta, \beta) \\
& \preccurlyeq \tau_{1}\left(\vee_{c b}(\alpha, \beta)\right) \bigvee_{c b}(\alpha, \beta)+\tau_{2}\left(\bigvee_{c b}(\alpha, \beta)\right) \bigvee_{c b}(\alpha, \beta) \\
& +\tau_{3}\left(\vee_{c b}(\alpha, \beta)\right) \vee_{c b}(\alpha, \beta)+\tau_{4}\left(\vee_{c b}(\alpha, \beta)\right) \vee_{c b}(\alpha, \beta) \\
& +\tau_{5}\left(\vee_{c b}(\alpha, \beta)\right) \vee_{c b}(\alpha, \beta) \\
& =\left(\tau_{1}\left(\bigvee_{c b}(\alpha, \beta)\right)+\tau_{2}\left(\bigvee_{c b}(\alpha, \beta)\right)+\tau_{3}\left(\bigvee_{c b}(\alpha, \beta)\right)+\tau_{4}\left(\bigvee_{c b}(\alpha, \beta)\right)\right. \\
& \left.+\tau_{5}\left(\vee_{c b}(\alpha, \beta)\right)\right) \vee_{c b}(\alpha, \beta) \\
& \preccurlyeq \frac{1}{2 s} \vee_{c b}(\alpha, \beta) \\
& \prec \curlyvee_{c b}(\alpha, \beta) \text {, }
\end{aligned}
$$

which implies that

$$
\left|\vee_{c b}(\alpha, \beta)\right|^{2}<\left|\vee_{c b}(\alpha, \beta)\right|
$$

which is impossible. Therefore, $\alpha=\beta$. Choose $\alpha_{1} \in \Theta$. Set

$$
\alpha_{2}=\Xi \alpha_{1}, \ldots, \alpha_{m+1}=\Xi \alpha_{m}=\Xi^{m+1} \alpha_{1} .
$$

Let $\vee_{m}=\vee_{c b}\left(\alpha_{m}, \Xi \alpha_{m}\right)$. If there exists $m \in \mathbb{N}$ such that $\vee_{m}=0$, the proof is complete. So, we presume that, for every $m \in \mathbb{N}, \quad 0 \prec \bigvee_{m}=\vee_{c b}\left(\alpha_{m}, \Xi \alpha_{m}\right)=\bigvee_{c b}\left(\alpha_{m}, \alpha_{m+1}\right)$. Therefore, we have

$$
\frac{1}{2 s} \bigvee_{c b}\left(\alpha_{m}, \Xi \alpha_{m}\right)<\bigvee_{c b}\left(\alpha_{m}, \Xi \alpha_{m}\right), \quad \forall m \in \mathbb{N} .
$$


From (2), we derive

$$
\begin{aligned}
& \bigvee_{c b}\left(\alpha_{m}, \Xi \alpha_{m}\right) \bigvee_{c b}\left(\Xi \alpha_{m}, \Xi^{2} \alpha_{m}\right) \leqslant \tau_{1}\left(\bigvee_{c b}\left(\alpha_{m}, \Xi \alpha_{m}\right)\right) \bigvee_{c b}\left(\alpha_{m}, \Xi \alpha_{m}\right) \\
& +\tau_{2}\left(\vee_{c b}\left(\alpha_{m}, \Xi \alpha_{m}\right)\right) \vee_{c b}\left(\alpha_{m}, \Xi^{2} \alpha_{m}\right) \\
& +\tau_{3}\left(\vee_{c b}\left(\alpha_{m}, \Xi \alpha_{m}\right)\right) \vee_{c b}\left(\Xi \alpha_{m}, \Xi \alpha_{m}\right) \\
& +\tau_{4}\left(\vee_{c b}\left(\alpha_{m}, \Xi \alpha_{m}\right)\right) \curlyvee_{c b}\left(\alpha_{m}, \Xi \alpha_{m}\right) \\
& +\tau_{5}\left(\vee_{c b}\left(\alpha_{m}, \Xi \alpha_{m}\right)\right) \vee_{c b}\left(\Xi \alpha_{m}, \Xi^{2} \alpha_{m}\right) \\
& \preccurlyeq \tau_{1}\left(\vee_{c b}\left(\alpha_{m}, \Xi \alpha_{m}\right)\right) \vee_{c b}\left(\alpha_{m}, \Xi \alpha_{m}\right) \\
& +s \tau_{2}\left(\vee_{c b}\left(\alpha_{m}, \Xi \alpha_{m}\right)\right) \vee_{c b}\left(\alpha_{m}, \Xi \alpha_{m}\right) \\
& +s \tau_{2}\left(\bigvee_{c b}\left(\alpha_{m}, \Xi \alpha_{m}\right)\right) \vee_{c b}\left(\Xi \alpha_{m}, \Xi^{2} \alpha_{m}\right) \\
& -\tau_{2}\left(\vee_{c b}\left(\alpha_{m}, \Xi \alpha_{m}\right)\right) \vee_{c b}\left(\Xi \alpha_{m}, \Xi \alpha_{m}\right) \\
& +s \tau_{3}\left(\vee_{c b}\left(\alpha_{m}, \Xi \alpha_{m}\right)\right) \vee_{c b}\left(\Xi \alpha_{m}, \alpha_{m}\right) \\
& +s \tau_{3}\left(\vee_{c b}\left(\alpha_{m}, \Xi \alpha_{m}\right)\right) \vee_{c b}\left(\alpha_{m}, \Xi \alpha_{m}\right) \\
& \text { - } \tau_{3}\left(\alpha_{m}, \Xi \alpha_{m}\right) \vee_{c b}\left(\alpha_{m}, \alpha_{m}\right) \\
& +\tau_{4}\left(\vee_{c b}\left(\alpha_{m}, \Xi \alpha_{m}\right)\right) \vee_{c b}\left(\alpha_{m}, \Xi \alpha_{m}\right) \\
& +\tau_{5}\left(\vee_{c b}\left(\alpha_{m}, \Xi \alpha_{m}\right)\right) \vee_{c b}\left(\Xi \alpha_{m}, \Xi^{2} \alpha_{m}\right) \\
& \leqslant \tau_{1}\left(\vee_{c b}\left(\alpha_{m}, \Xi \alpha_{m}\right)\right) \vee_{c b}\left(\alpha_{m}, \Xi \alpha_{m}\right) \\
& +s \tau_{2}\left(\vee_{c b}\left(\alpha_{m}, \Xi \alpha_{m}\right)\right) \vee_{c b}\left(\alpha_{m}, \Xi \alpha_{m}\right) \\
& +s \tau_{2}\left(\vee_{c b}\left(\alpha_{m}, \Xi \alpha_{m}\right)\right) \vee_{c b}\left(\Xi \alpha_{m}, \Xi^{2} \alpha_{m}\right) \\
& +s \tau_{3}\left(\vee_{c b}\left(\alpha_{m}, \Xi \alpha_{m}\right)\right) \vee_{c b}\left(\Xi \alpha_{m}, \alpha_{m}\right) \\
& +s \tau_{3}\left(\vee_{c b}\left(\alpha_{m}, \Xi \alpha_{m}\right)\right) \vee_{c b}\left(\alpha_{m}, \Xi \alpha_{m}\right) \\
& +\tau_{4}\left(\vee_{c b}\left(\alpha_{m}, \Xi \alpha_{m}\right)\right) \vee_{c b}\left(\alpha_{m}, \Xi \alpha_{m}\right) \\
& +\tau_{5}\left(\vee_{c b}\left(\alpha_{m}, \Xi \alpha_{m}\right)\right) \curlyvee_{c b}\left(\Xi \alpha_{m}, \Xi^{2} \alpha_{m}\right) \\
& =\left[\tau_{1}\left(\bigvee_{c b}\left(\alpha_{m}, \Xi \alpha_{m}\right)\right)+s \tau_{2}\left(\bigvee_{c b}\left(\alpha_{m}, \Xi \alpha_{m}\right)\right)\right. \\
& \left.+2 s \tau_{3}\left(\vee_{c b}\left(\alpha_{m}, \Xi \alpha_{m}\right)\right)+\tau_{4}\left(\vee_{c b}\left(\alpha_{m}, \Xi \alpha_{m}\right)\right)\right] \vee_{c b}\left(\alpha_{m}, \Xi \alpha_{m}\right) \\
& +\left[s \tau_{2}\left(\vee_{c b}\left(\alpha_{m}, \Xi \alpha_{m}\right)\right)+\tau_{5}\left(\vee_{c b}\left(\alpha_{m}, \Xi \alpha_{m}\right)\right)\right] \vee_{c b}\left(\Xi \alpha_{m}, \Xi^{2} \alpha_{m}\right) \text {, }
\end{aligned}
$$

and consequently,

$$
\begin{aligned}
& \vee_{m} \bigvee_{m+1} \prec {\left[\tau_{1}\left(\vee_{m}\right)+s \tau_{2}\left(\vee_{m}\right)+2 s \tau_{3}\left(\vee_{m}\right)+\tau_{4}\left(\vee_{m}\right)\right] \vee_{m} } \\
&+\left[s \tau_{2}\left(\vee_{m}\right)+\tau_{5}\left(\vee_{m}\right)\right] \vee_{m+1},
\end{aligned}
$$

which implies that

$$
\bigvee_{m+1} \prec \frac{\tau_{1}\left(\bigvee_{m}\right)+s \tau_{2}\left(\bigvee_{m}\right)+2 s \tau_{3}\left(\bigvee_{m}\right)+\tau_{4}\left(\bigvee_{m}\right)}{\bigvee_{m}-s \tau_{2}\left(\bigvee_{m}\right)-\tau_{5}\left(\bigvee_{m}\right)} \bigvee_{m} .
$$

From $\left(C_{3}\right)$, we obtain

$$
\begin{aligned}
\tau_{1}(v) & +2 s \tau_{2}(v)+2 s \tau_{3}(v)+\tau_{4}(v)+\tau_{5}(v) \prec 2 s\left[\tau_{1}(v)+\tau_{2}(v)+\tau_{3}(v)+\tau_{4}(v)+\tau_{5}(v)\right] \\
& <2 s \frac{1}{2 s^{2}} v=\frac{1}{s} v \prec v, \quad \forall v>0 .
\end{aligned}
$$

Consequently, we derive

$$
\frac{\tau_{1}(v)+s \tau_{2}(v)+2 s \tau_{3}(v)+\tau_{4}(v)}{v-s \tau_{2}(v)-\tau_{5}(v)} \prec 1, \quad \forall v>0 .
$$

Therefore, from (10), we obtain

$$
\vee_{c b}\left(\alpha_{m+1}, \Xi \alpha_{m+1}\right)=\vee_{m+1} \prec \bigvee_{m}=\bigvee_{c b}\left(\alpha_{m}, \Xi \alpha_{m}\right), \quad \forall m \in \mathbb{N},
$$

which implies that

$$
\left|\vee_{c b}\left(\alpha_{m+1}, \Xi \alpha_{m+1}\right)\right|<\left|\vee_{c b}\left(\alpha_{m}, \Xi \alpha_{m}\right)\right|, \quad \forall m \in \mathbb{N} .
$$

Consequently, Re.part $\left(\vee_{m+1}\right)<\operatorname{Re} \cdot \operatorname{part}\left(\vee_{m}\right)$ and Im.part $\left(\vee_{m+1}\right)<\operatorname{Im}$.part $\left(\vee_{m}\right)$. Therefore, $\left\{\operatorname{Re} \cdot \operatorname{part}\left(\vee_{m}\right)\right\}$ is a decreasing sequence of real numbers which is bounded from below. So, $\left\{\operatorname{Re}\right.$ part $\left.\left(\vee_{m}\right)\right\}$ converges to some point $\vee \in[0$, $\infty)$. Similarly, $\left\{\operatorname{Im}\right.$.part $\left.\left(\vee_{m}\right)\right\}$ converges to some point $\vee \in[0$, 
$\infty)$. Hence, $\left\{\bigvee_{m}=\bigvee_{c b}\left(\alpha_{m}, \Xi \alpha_{m}\right)\right\}$ converges to some point $\checkmark \in[0, \infty)$. If $\vee>0$, then using condition $\left(C_{1}\right)$, we obtain

$$
\begin{aligned}
& \checkmark=\lim _{m \longrightarrow \infty} \bigvee_{m+1}=\lim _{m \longrightarrow \infty} \sup \bigvee_{m+1} \\
& \preccurlyeq \lim _{m \longrightarrow \infty} \sup \frac{\tau_{1}\left(\bigvee_{m}\right)+s \tau_{2}\left(\bigvee_{m}\right)+2 s \tau_{3}\left(\bigvee_{m}\right)+\tau_{4}\left(\bigvee_{m}\right)}{\bigvee_{m}-s \tau_{2}\left(\bigvee_{m}\right)-\tau_{5}\left(\bigvee_{m}\right)} \bigvee_{m} \\
& =\frac{\tau_{1}(\curlyvee)+s \tau_{2}(\curlyvee)+2 s \tau_{3}(\curlyvee)+\tau_{4}(\curlyvee)}{\curlyvee-s \tau_{2}(\curlyvee)-\tau_{5}(\curlyvee)} \vee
\end{aligned}
$$

$\prec \curlyvee$,

which implies that $|\vee|<|\vee|$, which is impossible. Therefore,

$$
\lim _{m \longrightarrow \infty} \bigvee_{m}=\lim _{m \longrightarrow \infty} \bigvee_{c b}\left(\alpha_{m}, \Xi \alpha_{m}\right)=0 .
$$

Next, we prove that

$$
\lim _{m, r \longrightarrow \infty} \bigvee_{c b}\left(\alpha_{m}, \alpha_{r}\right)=0 .
$$

Suppose not, we assume that there exist $\varepsilon>0$ and sequence $\{\zeta(m)\}$ and $\{\xi(m)\}$ of natural numbers such that

$$
\begin{aligned}
\zeta(m) & >\xi(m)>m, \\
\vee_{c b}\left(\alpha_{\zeta(m)}, \alpha_{\xi(m)}\right) & \geqslant \varepsilon, \\
\vee_{c b}\left(\alpha_{\zeta(m)-1}, \alpha_{\xi(m)}\right) & <\mathcal{E}, \quad \forall n \in \mathbb{N} .
\end{aligned}
$$

Therefore, we derive

$$
\begin{aligned}
\varepsilon & \prec \bigvee_{c b}\left(\alpha_{\zeta(m)}, \alpha_{\xi(m)}\right) \prec s\left(\bigvee_{c b}\left(\alpha_{\zeta(m)}, \alpha_{\zeta(m)-1}\right)+\bigvee_{c b}\left(\alpha_{\zeta(m)-1}, \alpha_{\xi(m)}\right)\right) \\
& -\bigvee_{c b}\left(\alpha_{\zeta(m)-1}, \alpha_{\zeta(m)-1}\right) \\
& \prec s \vee_{c b}\left(\alpha_{\zeta(m)}, \alpha_{\zeta(m)-1}\right)+s \bigvee_{c b}\left(\alpha_{\zeta(m)-1}, \alpha_{\xi(m)}\right) \\
& \prec s \vee_{c b}\left(\alpha_{\zeta(m)}, \alpha_{\zeta(m)-1}\right)+s \mathcal{E} \\
& =s \vee_{c b}\left(\alpha_{\zeta(m)-1}, \Xi \alpha_{\zeta(m)-1}\right)+s \varepsilon .
\end{aligned}
$$

Using (16), we derive

$$
\varepsilon \prec \lim _{m \longrightarrow \infty} \inf _{c b}\left(\alpha_{\zeta(m)}, \alpha_{\xi(m)}\right) \prec \lim _{m \longrightarrow \infty} \sup _{c b}\left(\alpha_{\zeta(m)}, \alpha_{\xi(m)}\right) \prec s \varepsilon .
$$

From (16), there exists $M \in \mathbb{N}$ such that $(1 / 2 s) \vee_{c b}\left(\alpha_{\zeta(m)}\right.$, $\left.\Xi \alpha_{\zeta(m)}\right)<\mathcal{E}, \forall m>M$, and using (18), we derive

$$
\begin{array}{r}
\frac{1}{2 s} \bigvee_{c b}\left(\alpha_{\zeta(m)}, \Xi \alpha_{\zeta(m)}\right) \prec \bigvee_{c b}\left(\alpha_{\zeta(m)+1}, \alpha_{\xi(m)+1}\right) \\
=\bigvee_{c b}\left(\Xi \alpha_{\zeta(m)}, \Xi \alpha_{\xi(m)}\right), \quad \forall m>M .
\end{array}
$$

Therefore, from $\left(C_{3}\right)$, for every $m>M$, we obtain

$$
\begin{aligned}
& \vee_{c b}\left(\alpha_{\zeta(m)}, \alpha_{\xi(m)}\right) \vee_{c b}\left(\Xi \alpha_{\zeta(m)}, \Xi \alpha_{\xi(m)}\right) \leqslant \tau_{1}\left(\vee_{c b}\left(\alpha_{\zeta(m)}, \alpha_{\xi(m)}\right)\right) \vee_{c b}\left(\alpha_{\zeta(m)}, \alpha_{\xi(m)}\right) \\
& +\tau_{2}\left(\vee_{c b}\left(\alpha_{\zeta(m)}, \alpha_{\xi(m)}\right)\right) \vee_{c b}\left(\alpha_{\zeta(m)}, \Xi \alpha_{\xi(m)}\right) \\
& +\tau_{3}\left(\vee_{c b}\left(\alpha_{\zeta(m)}, \alpha_{\xi(m)}\right)\right) \vee_{c b}\left(\Xi \alpha_{\zeta(m)}, \alpha_{\xi(m)}\right) \\
& +\tau_{4}\left(\vee_{c b}\left(\alpha_{\zeta(m)}, \alpha_{\xi(m)}\right)\right) \vee_{c b}\left(\alpha_{\zeta(m)}, \Xi \alpha_{\zeta(m)}\right) \\
& +\tau_{5}\left(\vee_{c b}\left(\alpha_{\zeta(m)}, \alpha_{\xi(m)}\right)\right) \vee_{c b}\left(\alpha_{\xi(m)}, \Xi \alpha_{\xi(m)}\right) \\
& \leqslant \tau_{1}\left(\vee_{c b}\left(\alpha_{\zeta(m)}, \alpha_{\xi(m)}\right)\right) \vee_{c b}\left(\alpha_{\zeta(m)}, \alpha_{\xi(m)}\right) \\
& +\tau_{2}\left(\vee_{c b}\left(\alpha_{\zeta(m)}, \alpha_{\xi(m)}\right)\right) \vee_{c b}\left(\alpha_{\zeta(m)}, \Xi \alpha_{\xi(m)}\right) \\
& +\tau_{3}\left(\vee_{c b}\left(\alpha_{\zeta(m)}, \alpha_{\xi(m)}\right)\right) \vee_{c b}\left(\Xi \alpha_{\zeta(m)}, \alpha_{\xi(m)}\right) \\
& +s \tau_{4}\left(\vee_{c b}\left(\alpha_{\zeta(m)}, \alpha_{\xi(m)}\right)\right) \vee_{c b}\left(\alpha_{\zeta(m)}, \alpha_{\xi(m)}\right) \\
& +s \tau_{4}\left(\vee_{c b}\left(\alpha_{\zeta(m)}, \alpha_{\xi(m)}\right)\right) \vee_{c b}\left(\alpha_{\xi(m)}, \Xi \alpha_{\zeta(m)}\right) \\
& -\tau_{4}\left(\vee_{c b}\left(\alpha_{\zeta(m)}, \alpha_{\xi(m)}\right)\right) \vee_{c b}\left(\alpha_{\xi(m)}, \alpha_{\xi(m)}\right) \\
& +s \tau_{5}\left(\vee_{c b}\left(\alpha_{\zeta(m)}, \alpha_{\xi(m)}\right)\right) \vee_{c b}\left(\Xi \alpha_{\xi(m)}, \alpha_{\zeta(m)}\right) \\
& +s \tau_{5}\left(\vee_{c b}\left(\alpha_{\zeta(m)}, \alpha_{\xi(m)}\right)\right) \curlyvee_{c b}\left(\alpha_{\zeta(m)}, \alpha_{\xi(m)}\right) \\
& -\tau_{5}\left(\vee_{c b}\left(\alpha_{\zeta(m)}, \alpha_{\xi(m)}\right)\right) \vee_{c b}\left(\alpha_{\zeta(m)}, \alpha_{\zeta(m)}\right) \\
& \preccurlyeq \tau_{1}\left(\vee_{c b}\left(\alpha_{\zeta(m)}, \alpha_{\xi(m)}\right)\right) \vee_{c b}\left(\alpha_{\zeta(m)}, \alpha_{\xi(m)}\right) \\
& +\tau_{2}\left(\vee_{c b}\left(\alpha_{\zeta(m)}, \alpha_{\xi(m)}\right)\right) \vee_{c b}\left(\alpha_{\zeta(m)}, \Xi \alpha_{\xi(m)}\right) \\
& +\tau_{3}\left(\vee_{c b}\left(\alpha_{\zeta(m)}, \alpha_{\xi(m)}\right)\right) \vee_{c b}\left(\alpha_{\xi(m)}, \Xi \alpha_{\zeta(m)}\right) \\
& +s \tau_{4}\left(\vee_{c b}\left(\alpha_{\zeta(m)}, \alpha_{\xi(m)}\right)\right) \vee_{c b}\left(\alpha_{\zeta(m)}, \alpha_{\xi(m)}\right) \\
& +s \tau_{4}\left(\vee_{c b}\left(\alpha_{\zeta(m)}, \alpha_{\xi(m)}\right)\right) \vee_{c b}\left(\alpha_{\xi(m)}, \Xi \alpha_{\zeta(m)}\right) \\
& +s \tau_{5}\left(\vee_{c b}\left(\alpha_{\zeta(m)}, \alpha_{\xi(m)}\right)\right) \vee_{c b}\left(\Xi \alpha_{\xi(m)}, \alpha_{\zeta(m)}\right) \\
& +s \tau_{5}\left(\vee_{c b}\left(\alpha_{\zeta(m)}, \alpha_{\xi(m)}\right)\right) \vee_{c b}\left(\left(\alpha_{\zeta(m)}, \alpha_{\xi(m)}\right)\right. \\
& \preccurlyeq\left[\tau_{1}\left(\vee_{c b}\left(\alpha_{\zeta(m)}, \alpha_{\xi(m)}\right)\right)+s \tau_{4}\left(\vee_{c b}\left(\alpha_{\zeta(m)}, \alpha_{\xi(m)}\right)\right)+s \tau_{5}\left(\vee_{c b}\left(\alpha_{\zeta(m)}, \alpha_{\xi(m)}\right)\right)\right] \vee_{c b}\left(\alpha_{\zeta(m)}, \alpha_{\xi(m)}\right) \\
& +\left[\tau_{2}\left(\vee_{c b}\left(\alpha_{\zeta(m)}, \alpha_{\xi(m)}\right)\right)+s \tau_{5}\left(\vee_{c b}\left(\alpha_{\zeta(m)}, \alpha_{\xi(m)}\right)\right)\right] \vee_{c b}\left(\Xi \alpha_{\xi(m)}, \alpha_{\zeta(m)}\right) \\
& +\left[\tau_{3}\left(\vee_{c b}\left(\alpha_{\zeta(m)}, \alpha_{\xi(m)}\right)\right)+s \tau_{4}\left(\vee_{c b}\left(\alpha_{\zeta(m)}, \alpha_{\xi(m)}\right)\right)\right] \vee_{c b}\left(\alpha_{\xi(m)}, \Xi \alpha_{\zeta(m)}\right) .
\end{aligned}
$$


Hence, from $\left(C_{2}\right)$, we derive

$$
\begin{aligned}
\bigvee_{c b}\left(\alpha_{\zeta(m)}, \alpha_{\xi(m)}\right) \bigvee_{c b}\left(\Xi \alpha_{\zeta(m)}, \Xi \alpha_{\xi(m)}\right) \preccurlyeq & \frac{1}{2 s^{2}}\left[\bigvee_{c b}\left(\alpha_{\zeta(m)}, \alpha_{\xi(m)}\right)\right]^{2} \\
& +\frac{1}{2 s^{2}} \bigvee_{c b}\left(\alpha_{\zeta(m)}, \alpha_{\xi(m)}\right) \bigvee_{c b}\left(\Xi \alpha_{\zeta(m)}, \alpha_{\zeta(m)}\right) \\
& +\frac{1}{2 s^{2}} \bigvee_{c b}\left(\alpha_{\zeta(m)}, \alpha_{\xi(m)}\right) \vee_{c b}\left(\alpha_{\xi(m)}, \Xi \alpha_{\xi(m)}\right) .
\end{aligned}
$$

From (16)-(23), we obtain

$$
\begin{aligned}
\varepsilon^{2} & \prec \lim _{m \longrightarrow \infty} \sup \left[\vee_{c b}\left(\alpha_{\zeta(m)}, \alpha_{\xi(m)}\right) \vee_{c b}\left(\alpha_{\zeta(m)+1}, \alpha_{\xi(m)+1}\right)\right] \\
& \prec \lim _{m \longrightarrow \infty} \sup \left[\frac{1}{2 s^{2}}\left[\vee_{c b}\left(\alpha_{\zeta(m)}, \alpha_{\xi(m)}\right)\right]^{2} n+\frac{1}{2 s^{2}} \bigvee_{c b}\left(\alpha_{\zeta(m)}, \alpha_{\xi(m)}\right) \vee_{c b}\left(\Xi \alpha_{\zeta(m)}, \alpha_{\zeta(m)}\right) n\right. \\
& \left.\quad+\frac{1}{2 s^{2}} \vee_{c b}\left(\alpha_{\zeta(m)}, \alpha_{\xi(m)}\right) \vee_{c b}\left(\alpha_{\xi(m)}, \Xi \alpha_{\xi(m)}\right)\right] n \\
& \prec \frac{1}{2 s^{2}}(s \varepsilon)^{2}=\frac{1}{2} \varepsilon^{2} \prec \varepsilon^{2},
\end{aligned}
$$

which is impossible. Hence, $\lim _{m, r \rightarrow \infty} \vee_{c b}\left(\alpha_{m}, \alpha_{r}\right)=0$. By completeness of $\left(\Theta, \vee_{c b}\right)$, there exists $\alpha \in \Theta$ such that

$$
\bigvee_{c b}(\alpha, \alpha)=\lim _{m \longrightarrow \infty} \bigvee_{c b}\left(\alpha_{m}, \alpha\right)=\lim _{m, r \longrightarrow \infty} \bigvee_{c b}\left(\alpha_{m}, \alpha_{r}\right)=0 \text {. }
$$

We shall prove that, for every $m \in \mathbb{N}$,

$$
\frac{1}{2 s} \curlyvee_{c b}\left(\alpha_{m}, \Xi \alpha_{m}\right) \prec \bigvee_{c b}\left(\alpha_{m}, \alpha\right)
$$

or

$$
\frac{1}{2 s} \curlyvee_{c b}\left(\Xi \alpha_{m}, \Xi^{2} \alpha_{m}\right) \prec \bigvee_{c b}\left(\Xi \alpha_{m}, \alpha\right)
$$

Suppose not, we assume that there exists $q \in \mathbb{N}$ such that

$$
\begin{array}{r}
\frac{1}{2 s} \bigvee_{c b}\left(\alpha_{q}, \Xi \alpha_{q}\right) \geqslant \vee_{c b}\left(\alpha_{q}, \alpha\right), \\
\frac{1}{2 s} \curlyvee_{c b}\left(\Xi \alpha_{q}, \Xi^{2} \alpha_{q}\right) \geqslant \curlyvee_{c b}\left(\Xi \alpha_{q}, \alpha\right) .
\end{array}
$$

By the definition of complex partial $b$-metric space and (28), we derive

$$
\begin{aligned}
\bigvee_{c b}\left(\alpha_{q}, \Xi \alpha_{q}\right) & \prec s\left[\bigvee_{c b}\left(\alpha_{q}, \alpha\right)+\bigvee_{c b}\left(\alpha, \Xi \alpha_{q}\right)\right]-\bigvee_{c b}(\alpha, \alpha) \\
= & s \bigvee_{c b}\left(\alpha_{q}, \alpha\right)+s \bigvee_{c b}\left(\alpha, \Xi \alpha_{q}\right) \\
& \prec s \frac{1}{2 s} \bigvee_{c b}\left(\alpha_{q}, \Xi \alpha_{q}\right)+s \frac{1}{2 s} \bigvee_{c b}\left(\Xi \alpha_{q}, \Xi^{2} \alpha_{q}\right) \\
& \prec \frac{1}{2} \bigvee_{c b}\left(\alpha_{q}, \Xi \alpha_{q}\right) \\
& +\frac{1}{2} \bigvee_{c b}\left(\alpha_{q}, \Xi \alpha_{q}\right) \\
= & \vee_{c b}\left(\alpha_{q}, \Xi \alpha_{q}\right),
\end{aligned}
$$

which is impossible. Hence, (25) and (26) holds. From (25), we obtain

$$
\begin{aligned}
& \vee_{c b}\left(\alpha_{m}, \alpha\right) \vee_{c b}\left(\Xi \alpha_{m}, \Xi \alpha\right) \preccurlyeq \tau_{1}\left(\vee_{c b}\left(\alpha_{m}, \alpha\right)\right) \vee_{c b}\left(\alpha_{m}, \alpha\right) \\
& +\tau_{2}\left(\vee_{c b}\left(\alpha_{m}, \alpha\right)\right) \vee_{c b}\left(\alpha_{m}, \Xi \alpha\right) \\
& +\tau_{3}\left(\vee_{c b}\left(\alpha_{m}, \alpha\right)\right) \vee_{c b}\left(\Xi \alpha_{m}, \alpha\right) \\
& +\tau_{4}\left(\vee_{c b}\left(\alpha_{m}, \alpha\right)\right) \bigvee_{c b}\left(\alpha_{m}, \Xi \alpha_{m}\right) \\
& +\tau_{5}\left(\vee_{c b}\left(\alpha_{m}, \alpha\right)\right) \vee_{c b}(\alpha, \Xi \alpha) \\
& \preccurlyeq \tau_{1}\left(\vee_{c b}\left(\alpha_{m}, \alpha\right)\right) \vee_{c b}\left(\alpha_{m}, \alpha\right) \\
& +s \tau_{2}\left(\vee_{c b}\left(\alpha_{m}, \alpha\right)\right) \vee_{c b}\left(\alpha_{m}, \Xi \alpha_{m}\right) \\
& +s \tau_{2}\left(\vee_{c b}\left(\alpha_{m}, \alpha\right)\right) \vee_{c b}\left(\Xi \alpha_{m}, \Xi \alpha\right) \\
& +\tau_{3}\left(\vee_{c b}\left(\alpha_{m}, \alpha\right)\right) \vee_{c b}\left(\Xi \alpha_{m}, \alpha\right) \\
& +\tau_{4}\left(\vee_{c b}\left(\alpha_{m}, \alpha\right)\right) \vee_{c b}\left(\alpha_{m}, \Xi \alpha_{m}\right)
\end{aligned}
$$




$$
\begin{aligned}
+ & +s \tau_{5}\left(\vee_{c b}\left(\alpha_{m}, \alpha\right)\right) \vee_{c b}\left(\alpha, \Xi \alpha_{m}\right) \\
& +s \tau_{5}\left(( \vee _ { c b } ( \alpha _ { m } , \alpha ) ) \vee _ { c b } \left(\left(\Xi \alpha_{m}, \Xi \alpha\right)\right.\right. \\
\leqslant & \tau_{1}\left(\vee_{c b}\left(\alpha_{m}, \alpha\right)\right) \vee_{c b}\left(\alpha_{m}, \alpha\right) \\
+ & {\left[s \tau_{2}\left(\vee_{c b}\left(\alpha_{m}, \alpha\right)\right)+\tau_{4}\left(\vee_{c b}\left(\alpha_{m}, \alpha\right)\right)\right] \vee_{c b}\left(\alpha_{m}, \Xi \alpha_{m}\right) } \\
+ & {\left[s \tau_{2}\left(\vee_{c b}\left(\alpha_{m}, \alpha\right)\right)+s \tau_{5}\left(\vee_{c b}\left(\alpha_{m}, \alpha\right)\right)\right] \vee_{c b}\left(\Xi \alpha_{m}, \Xi \alpha\right) } \\
+ & {\left[\tau_{3}\left(\vee_{c b}\left(\alpha_{m}, \alpha\right)\right)+s \tau_{5}\left(\vee_{c b}\left(\alpha_{m}, \alpha\right)\right)\right] \vee_{c b}\left(\Xi \alpha_{m}, \alpha\right) . }
\end{aligned}
$$

From (12), we derive

$$
\begin{aligned}
\vee_{c b}\left(\Xi \alpha_{m}, \Xi \alpha\right) \leqslant & \vee_{c b}\left(\alpha_{m}, \alpha\right)+\vee_{c b}\left(\alpha_{m}, \Xi \alpha_{m}\right) \\
& +\vee_{c b}\left(\Xi \alpha_{m}, \Xi \alpha\right)+\bigvee_{c b}\left(\Xi \alpha_{m}, \alpha\right) .
\end{aligned}
$$

Using (25) and (26), we derive

$$
\lim _{m \longrightarrow \infty} \bigvee_{c b}\left(\Xi \alpha_{m}, \Xi \alpha\right)=0 \text {. }
$$

Since

$$
\begin{aligned}
\vee_{c b}(\alpha, \Xi \alpha)<s\left[\bigvee_{c b}\left(\alpha, \Xi \alpha_{m}\right)\right. & \left.+\vee_{c b}\left(\Xi \alpha_{m}, \Xi \alpha\right)\right]-\vee_{c b}\left(\Xi \alpha_{m}, \Xi \alpha_{m}\right) \\
& \prec s\left[\curlyvee_{c b}\left(\alpha, \Xi \alpha_{m}\right)+\vee_{c b}\left(\Xi \alpha_{m}, \Xi \alpha\right)\right] \\
& =s \vee_{c b}\left(\alpha, \alpha_{m+1}\right)+s \vee_{c b}\left(\Xi \alpha_{m}, \Xi \alpha\right),
\end{aligned}
$$

using (25) and (32), we get $\alpha=\Xi \alpha$. From (27), we obtain

$$
\begin{aligned}
& \vee_{c b}\left(\Xi \alpha_{m}, \alpha\right) \vee_{c b}\left(\Xi^{2} \alpha_{m}, \Xi \alpha\right) \leqslant \tau_{1}\left(\vee_{c b}\left(\Xi \alpha_{m}, \alpha\right)\right) \vee_{c b}\left(\Xi \alpha_{m}, \alpha\right) \\
& +\tau_{2}\left(\vee_{c b}\left(\Xi \alpha_{m}, \alpha\right)\right) \vee_{c b}\left(\Xi \alpha_{m}, \Xi \alpha\right) \\
& +\tau_{3}\left(\vee_{c b}\left(\Xi \alpha_{m}, \alpha\right)\right) \vee_{c b}\left(\Xi^{2} \alpha_{m}, \alpha\right) \\
& +\tau_{4}\left(\vee_{c b}\left(\Xi \alpha_{m}, \alpha\right)\right) \vee_{c b}\left(\Xi \alpha_{m}, \Xi^{2} \alpha_{m}\right) \\
& +\tau_{5}\left(\vee_{c b}\left(\Xi \alpha_{m}, \alpha\right)\right) \curlyvee_{c b}(\alpha, \Xi \alpha) \\
& \leqslant \tau_{1}\left(\vee_{c b}\left(\Xi \alpha_{m}, \alpha\right)\right) \vee_{c b}\left(\Xi \alpha_{m}, \alpha\right) \\
& +s \tau_{2}\left(\vee_{c b}\left(\Xi \alpha_{m}, \alpha\right)\right) \vee_{c b}\left(\Xi \alpha_{m}, \Xi^{2} \alpha_{m}\right) \\
& +s \tau_{2}\left(\vee_{c b}\left(\Xi \alpha_{m}, \alpha\right)\right) \vee_{c b}\left(\Xi^{2} \alpha_{m}, \Xi \alpha\right) \\
& -\tau_{2}\left(\vee_{c b}\left(\Xi \alpha_{m}, \alpha\right)\right) \vee_{c b}\left(\Xi^{2} \alpha_{m}, \Xi^{2} \alpha_{m}\right) \\
& +\tau_{3}\left(\vee_{c b}\left(\Xi \alpha_{m}, \alpha\right)\right) \vee_{c b}\left(\Xi^{2} \alpha_{m}, \alpha\right) \\
& +\tau_{4}\left(\vee_{c b}\left(\Xi \alpha_{m}, \alpha\right)\right) \vee_{c b}\left(\Xi \alpha_{m}, \Xi^{2} \alpha_{m}\right) \\
& +s \tau_{5}\left(\vee_{c b}\left(\Xi \alpha_{m}, \alpha\right)\right) \vee_{c b}\left(\alpha, \Xi^{2} \alpha_{m}\right) \\
& +s \tau_{5}\left(\vee_{c b}\left(\Xi \alpha_{m}, \alpha\right)\right) \vee_{c b}\left(\Xi^{2} \alpha_{m}, \Xi \alpha\right) \\
& \tau_{5}\left(\bigvee_{c b}\left(\Xi \alpha_{m}, \alpha\right)\right) \bigvee_{c b}\left(\Xi^{2} \alpha_{m}, \Xi^{2} \alpha_{m}\right) \\
& \preccurlyeq \tau_{1}\left(\vee_{c b}\left(\Xi \alpha_{m}, \alpha\right)\right) \vee_{c b}\left(\Xi \alpha_{m}, \alpha\right) \\
& +s \tau_{2}\left(\vee_{c b}\left(\Xi \alpha_{m}, \alpha\right)\right) \vee_{c b}\left(\Xi \alpha_{m}, \Xi^{2} \alpha_{m}\right) \\
& +s \tau_{2}\left(\vee_{c b}\left(\Xi \alpha_{m}, \alpha\right)\right) \vee_{c b}\left(\Xi^{2} \alpha_{m}, \Xi \alpha\right) \\
& +\tau_{3}\left(\vee_{c b}\left(\Xi \alpha_{m}, \alpha\right)\right) \curlyvee_{c b}\left(\Xi^{2} \alpha_{m}, \alpha\right) \\
& +\tau_{4}\left(\vee_{c b}\left(\Xi \alpha_{m}, \alpha\right)\right) \curlyvee_{c b}\left(\Xi \alpha_{m}, \Xi^{2} \alpha_{m}\right) \\
& +s \tau_{5}\left(\vee_{c b}\left(\Xi \alpha_{m}, \alpha\right)\right) \vee_{c b}\left(\alpha, \Xi^{2} \alpha_{m}\right) \\
& +s \tau_{5}\left(\vee_{c b}\left(\Xi \alpha_{m}, \alpha\right)\right) \vee_{c b}\left(\Xi^{2} \alpha_{m}, \Xi \alpha\right) \\
& \preccurlyeq \tau_{1}\left(\vee_{c b}\left(\Xi \alpha_{m}, \alpha\right)\right) \vee_{c b}\left(\Xi \alpha_{m}, \alpha\right) \\
& +\left[s \tau_{2}\left(\vee_{c b}\left(\Xi \alpha_{m}, \alpha\right)\right)+\tau_{4}\left(\vee_{c b}\left(\Xi \alpha_{m}, \alpha\right)\right)\right] \vee_{c b}\left(\Xi \alpha_{m}, \Xi^{2} \alpha_{m}\right) \\
& +\left[s \tau_{2}\left(\vee_{c b}\left(\Xi \alpha_{m}, \alpha\right)\right)+s \tau_{5}\left(\vee_{c b}\left(\Xi \alpha_{m}, \alpha\right)\right)\right] \vee_{c b}\left(\Xi^{2} \alpha_{m}, \Xi \alpha\right) \\
& +\left[\tau_{3}\left(\vee_{c b}\left(\Xi \alpha_{m}, \alpha\right)\right)+s \tau_{5}\left(\vee_{c b}\left(\Xi \alpha_{m}, \alpha\right)\right)\right] \vee_{c b}\left(\Xi^{2} \alpha_{m}, \alpha\right),
\end{aligned}
$$


which means that

$$
\begin{aligned}
\vee_{c b}\left(\Xi^{2} \alpha_{m}, \Xi \alpha\right)<\frac{\tau_{1}\left(\Xi \alpha_{m}, \alpha\right) \vee_{c b}\left(\Xi \alpha_{m}, \alpha\right)}{\vee_{c b}\left(\Xi \alpha_{m}, \alpha\right)-s \tau_{2}\left(\vee_{c b}\left(\Xi \alpha_{m}, \alpha\right)\right)-s \tau_{5}\left(\vee_{c b}\left(\Xi \alpha_{m}, \alpha\right)\right)} \\
+\frac{\left(s \tau_{2}\left(\vee_{c b}\left(\Xi \alpha_{m}, \alpha\right)\right)+\tau_{4}\left(\vee_{c b}\left(\Xi \alpha_{m}, \alpha\right)\right)\right) \vee_{c b}\left(\Xi \alpha_{m}, \Xi^{2} \alpha\right)}{\vee_{c b}\left(\Xi \alpha_{m}, \alpha\right)-s \tau_{2}\left(\vee_{c b}\left(\Xi \alpha_{m}, \alpha\right)\right)-s \tau_{5}\left(\vee_{c b}\left(\Xi \alpha_{m}, \alpha\right)\right)} \\
+\frac{\left(\tau_{3}\left(\vee_{c b}\left(\Xi \alpha_{m}, \alpha\right)\right)+s \tau_{5}\left(\vee_{c b}\left(\Xi \alpha_{m}, \alpha\right)\right)\right) \vee_{c b}\left(\Xi^{2} \alpha_{m}, \alpha\right)}{\bigvee_{c b}\left(\Xi \alpha_{m}, \alpha\right)-s \tau_{2}\left(\vee_{c b}\left(\Xi \alpha_{m}, \alpha\right)\right)-s \tau_{5}\left(\vee_{c b}\left(\Xi \alpha_{m}, \alpha\right)\right)} .
\end{aligned}
$$

From (12) and (35), we derive that

$$
\vee_{c b}(r, j)=\max \{r, j\}^{2}+|r-j|^{2}+i\left(\max \{r, j\}^{2}+|r-j|^{2}\right) .
$$

$0<\curlyvee_{c b}\left(\Xi^{2} \alpha_{m}, \Xi \alpha\right)<\curlyvee_{c b}\left(\Xi \alpha_{m}, \alpha\right)+\curlyvee_{c b}\left(\Xi \alpha_{m}, \Xi^{2} \alpha\right)+\curlyvee_{c b}\left(\alpha, \Xi^{2} \alpha_{m}\right)$.

Using (16) and (25), we derive

$$
\lim _{m \longrightarrow \infty} \bigvee_{c b}\left(\Xi^{2} \alpha_{m}, \Xi \alpha\right)=0
$$

Since

$$
\begin{aligned}
\vee_{c b}(\alpha, \Xi \alpha)< & s\left[\vee_{c b}\left(\alpha, \Xi^{2} \alpha_{m}\right)+\vee_{c b}\left(\Xi^{2} \alpha_{m}, \Xi \alpha\right)\right] \\
& -\vee_{c b}\left(\Xi^{2} \alpha_{m}, \Xi^{2} \alpha_{m}\right) \\
& <s\left[\vee_{c b}\left(\alpha, \Xi^{2} \alpha_{m}\right)+\vee_{c b}\left(\Xi^{2} \alpha_{m}, \Xi \alpha\right)\right],
\end{aligned}
$$

using (25) and (37), we get $\alpha=\Xi \alpha$.
Define a mapping $\Xi$ on $\Theta$ by

$$
-10 p t \Xi(r)= \begin{cases}2, & r \in \mathbb{N}, \\ 8 r+\frac{1}{j+3}, & r \in\left\{r+\frac{1}{j+3}: r, j \in \mathbb{N}\right\} .\end{cases}
$$

Then, $\Xi$ satisfies in the assumption of Theorem 2.

Proof. It is clear that $\left(\Xi, \vee_{c b}\right)$ is a complete complex partial $b$-metric space with coefficient $s=2^{2}$ and 2 is a unique fixed point of $\Xi$. Let $j<r$ :

Example 2. Let $\Theta=\mathbb{N} \cup\{r+(1 / j+3): r, j \in \mathbb{N}\}$. Define $\vee_{c b}: \Theta \times \Theta \longrightarrow \mathbb{C}^{+}$by

$$
\begin{aligned}
\frac{1}{2} \bigvee_{c b}\left(r+\frac{1}{j+3}, \Xi\left(r+\frac{1}{j+3}\right)\right)= & \frac{1}{2} \bigvee_{c b}\left(r+\frac{1}{j+3}, 8 r+\frac{1}{j+3}\right) \\
= & \frac{1}{2}\left(\max \left\{r+\frac{1}{j+3}, 8 r+\frac{1}{j+3}\right\}^{2}\right. \\
& +\left|r+\frac{1}{j+3}-8 r-\frac{1}{j+3}\right|^{2} \\
& \left.+i\left(\max \left\{r+\frac{1}{j+3}, 8 r+\frac{1}{j+3}\right\}^{2}+\left|r+\frac{1}{j+3}-8 r-\frac{1}{j+3}\right|^{2}\right)\right) \\
= & \frac{1}{2}\left(\left(8 r+\frac{1}{j+3}\right)^{2}+49 r^{2}+i\left(8 r+\frac{1}{j+3}\right)^{2}+49 r^{2}\right) \\
= & \frac{1}{2}\left(113 r^{2}+\frac{1}{(j+3)^{2}}+16 \frac{r}{j+3}+i\left(113 r^{2}+\frac{1}{(j+3)^{2}}+16 \frac{r}{j+3}\right)\right), \\
\bigvee_{c b}\left(r+\frac{1}{j+3}, j+\frac{1}{j+3}\right)= & \max \left\{r+\frac{1}{j+3}, j+\frac{1}{j+3}\right\}^{2}+\left|r+\frac{1}{j+3}-j-\frac{1}{j+3}\right|^{2}
\end{aligned}
$$




$$
\begin{aligned}
& +i\left(\max \left\{r+\frac{1}{j+3}, j+\frac{1}{j+3}\right\}^{2}+\left|r+\frac{1}{j+3}-j-\frac{1}{j+3}\right|^{2}\right) \\
= & \left(r+\frac{1}{j+3}\right)^{2}+|r-j|^{2}+i\left(\left(r+\frac{1}{j+3}\right)^{2}+|r-j|^{2}\right) \\
\prec & r^{2}+\frac{1}{(j+3)^{2}}+\frac{2 r}{j+3}+(|r|-|j|)^{2} \\
& +i\left(r^{2}+\frac{1}{(j+3)^{2}}+\frac{2 r}{j+3}+(|r|-|j|)^{2}\right) \\
\prec & r^{2}+\frac{1}{(j+3)^{2}}+\frac{2 r}{j+3}+(|r|+|j|)^{2} \\
& +i\left(r^{2}+\frac{1}{(j+3)^{2}}+\frac{2 r}{j+3}+(|r|+|j|)^{2}\right) .
\end{aligned}
$$

So, for $j<r, \quad(1 / 2) \bigvee_{c b}(r+(1 / j+3), \Xi(r+(1 / j+3)))$ $k \vee_{c b}(r+(1 / j+3), j+(1 / j+3))$. Therefore, $\Xi$ satisfies all the conditions of Theorem 2 .

3.1. Nonlinear Integral Equations. In this section, we prove the existence and uniqueness of a solution for nonlinear Fredholm integral equations by using Theorem 2 .

Theorem 3. Consider the nonlinear Fredholm integral equation:

$$
r(t)=j(t)+\int_{a}^{b} K(t, s, r(s)) \mathrm{d} s,
$$

where $a, b \in \mathbb{R}$ with $a<b$ and $j:[a, b] \longrightarrow \mathbb{R}$ and $K:[a, b]^{2} \times \mathbb{R} \longrightarrow \mathbb{R}$ are given continuous mappings. Suppose that the following condition holds:

(a) The mapping $\Xi: C[a, b] \longrightarrow C[a, b]$ defined by $(\Xi r)(t)=j(t)+\int_{a}^{b} K(t, s, r(s)) d s$ for all $r \in C[a, b]$ and $t \in[a, b]$.

Then, the nonlinear integral equation (42) has a unique solution.

Proof. Let $\Theta=C[a, b]$. Clearly, $\Theta$ with the complex partial $b$-metric $\vee_{c b}: \Theta \times \Theta \longrightarrow C^{+}$given by

$$
\begin{aligned}
\vee_{c b}(r(t), v(t))= & \max \{r(t), v(t)\}^{2} \\
& +i \max \{r(t), v(t)\}^{2}, \quad t \in[a, b],
\end{aligned}
$$

for all $r, v \in \Theta$ is a complete complex partial $b$-metric space. Without loss generality, we may assume that

$$
r \prec v \Leftrightarrow r(t) \leq v(t), \quad \forall t \in[a, b] .
$$

Now,

$$
\begin{array}{r}
\frac{1}{2 s}\left(\left(j(t)+\int_{a}^{b} K(t, s, v(s))\right)^{2}+i\left(\left(j(t)+\int_{a}^{b} K(t, s, v(s))\right)^{2}\right)\right) \\
<\left(j(t)+\int_{a}^{b} K(t, s, v(s))\right)^{2}+i\left(\left(j(t)+\int_{a}^{b} K(t, s, v(s))\right)^{2}\right) .
\end{array}
$$

Therefore,

$$
\frac{1}{2 s} \curlyvee_{c b}(v(t), \Xi v(t)) \prec \curlyvee_{c b}(r(t), v(t))
$$

Hence, $\Xi$ satisfies all the conditions of Theorem 2 .

\section{Conclusion}

In the present work, we presented a new fixed point theorem for self-mappings defined on complex partial $b$-metric. We illustrated our main theorem by an example and showed, moreover, that the main theorem can be easily used to solve a nonlinear integral equation.

\section{Data Availability}

No data were used in this study.

\section{Conflicts of Interest}

The authors declare that they have no conflicts of interest.

\section{References}

[1] I. A. Bakhtin, "The contraction mappings principle in quasimetric spaces," Functional Analysis, vol. 30, pp. 26-37, 1989, (Russian).

[2] S. Czerwick, "Contraction mappings in b-metric spaces," Acta Mathematica et Informatica Universitatis Ostraviensis, vol. 1, pp. 5-11, 1993.

[3] A. Azam, B. Fisher, and M. Khan, "Common fixed point theorems in complex valued metric spaces," Numerical 
Functional Analysis and Optimization, vol. 32, no. 3, pp. 243-253, 2011.

[4] K. P. R. Rao, P. R. Swamy, and J. R. Prasad, "A common fixed point theorem in complex valued $b$-metric spaces," Bulletin of Mathematics and Statistics Research, vol. 1, no. 1, 2013.

[5] P. Dhivya and M. Marudai, "Common fixed point theorems for mappings satisfying a contractive condition of rational expression on a ordered complex partial metric space," Cogent Mathematics, vol. 4, no. 1, Article ID 1389622, 2017.

[6] M. Gunaseelan, "Generalized fixed point theorems on complex partial b-metric space," International Journal of Research and Analytical Reviews, vol. 6, no. 2, 2019.

[7] A. Latif, T. Nazir, and M. Abbas, "Stability of fixed points in generalized metric spaces," Journal of Nonlinear Functional Analysis, vol. 2, pp. 287-294, 2018.

[8] A. Deepmala and H. K. Pathak, "A study on some problems on existence of solutions for nonlinear functional-integral equations," Acta Mathematica Scientia, vol. 33, no. 5, pp. 1305-1313, 2013.

[9] A. Deepmala, "Study on fixed point theorems for nonlinear contractions and its applications," Ph.D. Thesis, Ravishankar Shukla University, Chhatisgarh, India, 2014.

[10] F. Gu and W. Shatanawi, "Some new results on common coupled fixed points of two hybrid pairs of mappings in partial metric spaces," Journal of Nonlinear Functional Analysis, vol. 2019, p. 13, 2019.

[11] H. K. Pathak and A. Deepmala, "Common fixed point theorems for PD-operator pairs under relaxed conditions with applications," Journal of Computational and Applied Mathematics, vol. 239, pp. 103-113, 2013.

[12] M. Abbas, I. Beg, and B. T. Leyew, "Common fixed points of $(\mathrm{R}, \alpha)$-generalized rational multivalued contractions in R-complete $b$-metric spaces," Communications in Optimization Theory, vol. 2019, p. 14, 2019.

[13] Y. U. Gaba, "Fixed points on partial metric type spaces," Research Fixed Point Theory Appl.vol. 2019, Article ID 2019009, 21 pages, 2019.

[14] M. Aslantas, H. Sahin, and D. Turkoglu, "Some Caristi type fixed point theorems," The Journal of Analysis, vol. 29, pp. 1-15, 2020.

[15] H. Sahin, M. Aslantas, and I. Altun, "Feng-Liu type approach to best proximity point results for multivalued mappings," Journal of Fixed Point Theory and Applications, vol. 22, no. 1, p. $11,2020$.

[16] I. Altun, M. Aslantas, and H. Sahin, "Best proximity point results for p-proximal contractions," Acta Mathematica Hungarica, vol. 162, no. 2, pp. 393-402, 2020.

[17] M. Aslantas, H. Sahin, and I. Altun, "Best proximity point theorems for cyclic p-contractions with some consequences and applications," Nonlinear Analysis: Modelling and Control, vol. 26, no. 1, pp. 113-129, 2021.

[18] Y. U. Gaba, "Metric type spaces and $\lambda$-sequences," Quaestiones Mathematicae, vol. 40, no. 1, pp. 49-55, 2017.

[19] M. U. Ali, H. Aydi, and M. Alansari, "New generalizations of set valued interpolative Hardy-Rogers type contractions in b-metric spaces," Journal of Function Spaces, vol. 2021, Article ID 6641342, 8 pages, 2021.

[20] K. Javed, H. Aydi, F. Uddin, and M. Arshad, "On orthogonal partial b-metric spaces with an application," Journal of Mathematics, vol. 2021, Article ID 6692063, 7 pages, 2021.

[21] N. Mlaiki, N. Souayah, T. Abdeljawad, and H. Aydi, "A new extension to the controlled metric type spaces endowed with a graph," Advances in Difference Equations, vol. 2021, p. 94, 2021.
[22] M. Gunaseelan, L. N. Mishra, V. N. Mishra, I. A. Baloch, and M. De La Sen, "Application to coupled fixed-point theorems on complex partial b-metric space," Journal of Mathematics, vol. 2020, Article ID 8881859, 11 pages, 2020.

[23] A. Lemma maria prakasam and M. Gunaseelan, "Common fixed point theorems using CLR and EA properties in complex partial b-metric space," Advances in Mathematics: Scientific Journal, vol. 9, no. 5, pp. 2773-2790, 2020. 